\title{
Analytical Solution of Stiffness for a Corner-Fillet Leaf-Spring Type Flexure Hinge with a Long Fatigue Life
}

\author{
Li Rui-qi, Wu Bai-sheng, Chen Xin, Yang Zhi-jun
}

Micro and Nano Processing Equipment and Technology Key Laboratory of Guangdong Province, Guangdong University of Technology, Guangzhou, China

Email address:

1930516@qq.com(Li Rui-qi),wubs@gdut.edu.cn(Wu Bai-sheng),yangzj@gdut.edu.cn (Yang Zhi-jun),chenx@gdut.edu.cn(Chen Xin)

\section{To cite this article:}

Li Rui-qi, Wu Bai-sheng, Chen Xin, Yang Zhi-jun. Analytical Solution of Stiffness for a Corner-Fillet Leaf-Spring Type Flexure Hinge with a Long Fatigue Life. International Journal of Mechanical Engineering and Applications. Vol. 6, No. 3, 2018, pp. 64-72.

doi: 10.11648/j.ijmea.20180603.14

Received: May 17, 2018; Accepted: June 5, 2018; Published: June 29, 2018

\begin{abstract}
Flexure hinges as the displacement guiding and amplifying mechanism or sensing component are widely used for micro-actuators and sensors. However, the existing flexure hinges, leaf-spring or notch type, cause serious stress concentration which severely weaken the fatigue life of compliance mechanism. Therefore, developing long fatigue life flexure hinges is very important for high working frequency actuators and sensors, such as fast-tool-servo. Corner-fillet leaf-spring type flexure hinge could provide large displacement with lower stress. Stiffness expressions of it with both fixed-fixed and fixed-guided boundary conditions are derived by using Castigliano's theorem. The main influence factors for stress concentration are investigated and the formulas of stress concentration factor are obtained in terms of ratio of fillet radius to the minimum thickness. These analytical formulas have been verified by comparing with finite element analysis (FEA) results. Stress-life method is chosen to research the influence of fillet radius on fatigue life and the results indicate fillet radius can improve fatigue life of flexure hinge effectively. The proposed analytical solution is the fundamental of optimal design of a leaf-spring type flexure hinge based mechanism with fatigue life constraints.
\end{abstract}

Keywords: Stiffness, Stress Concentration, Corner-Fillet, Flexure Hinge, Long Fatigue Life

\section{Introduction}

Compliant mechanisms are widely used in micro actuator [1-3], micromanipulator [4], displacement amplifier [5], micro/nano positioning stages [6-9], accelerometers [10-12] and sensors [13, 14]. For micro/nano position stage, large stork and accurate displacement output, which make the displacement amplifying and guiding mechanisms become indispensable parts are two important goals to be achieved [15, 16]. Better sensitivity and faster response also make compliance mechanisms important to the sensors. As the basic elements of compliance mechanisms, flexure hinges are mainly divided into two types, notch type and leaf-spring (shown in Figure 1). Notch type flexure hinge is a relative rigid part, with different shapes include conic-section [17, 18], elliptical arc [19], V-shaped [20] and so on. Offering higher rotational accuracy, notch type flexure hinges are utilized for angular output and displacement amplification. Leaf-spring type flexure hinges can deform as a whole part, which are commonly used for large stroke displacement guidance and sensing elements of sensors.

However, stress concentration often occurs to both leaf-spring type flexure hinges and notch type flexure hinges, which produce high stress level when used for large displacement system. High stress level can lead to a failure of flexure hinge and even the entire compliant mechanisms under dynamic loading conditions [21, 22]. As actuators and sensors working at higher frequency, the fatigue problem becomes extremely important. Therefore, long fatigue life need to be considered in the flexure hinge design. Due to the large displacement output and its popular used in actuators and sensors, corner-fillet leaf-spring type flexure hinges(CFLSFH) are proposed for long fatigue life and large displacement in this paper, and the key work is to develop an analytic expression of the stiffness, and determine an optimal ratio of radium to the minimum thickness under fatigue life constraint. 

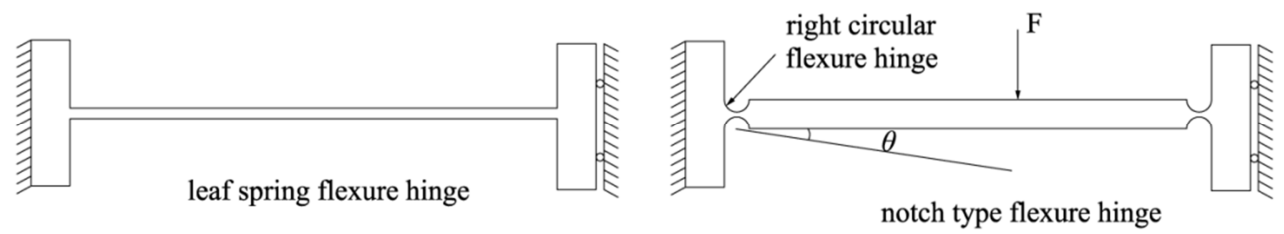

Figure 1. Existing flexure hinges.

Numerous studies have been made for the design of flexure hinges and compliant mechanism [23-27]. In those literatures, notch type flexure hinges are considered as fixed-free beams, and leaf-spring type flexure hinges are more easily found in fixed-fixed and fixed-guided condition and its design formulas are easily obtained for constant cross-section beam model. Although CFLSFHs are considered as leaf spring for simple design when fillet radius is small, the assumption is inaccuracy for large fillet radius CFLSFHs. CFLSFHs are cross-section varying beams, which is far more difficult to obtain the analytic solution of stiffness for either fixed-fixed or fixed-guided condition. To solve this problem, similar researches have been reported by using Castiliagno's theorem [28] and inverse conformal mapping [23]. Although the finite element method can analyze the stiffness and fatigue life of a flexure hinge efficiently, it cannot deal with the topology and size optimization simultaneously. Recently, the authors [29] presented a simultaneous optimal design method of topology and size under stiffness and frequency constraints using analytical solution of the equivalent stiffness and mass of flexure hinge. Therefore, the analytical solution of CFLSFHs should be figure out for the further optimizing of guiding mechanism under fatigue life constraints.
In addition, how to design an appropriate fillet to reduce stress and ensure good flexibility and enough deformation area of CFLSFH is another problem need to be solved, which is illustrated in Figure 2 (a) and (b). Although the shape of CFLSFH is similar to corner fillet flexure hinge, their constraints are different. Corner fillet flexure hinges are commonly designed as cantilever beam [28, 30-31], which is shown in Figure 2 (c). So their achievements can't be used for the design of CFLSFH directly. In this paper, the static models of CFLSFH under fixed-guided and fixed-fixed constraint are built and their stiffness equations are deduced based on force method and Castiliagno's theorem. The curves of stiffness variation with dimensionless geometric parameters $a$ (ratio of fillet radius to minimum thickness) and $b$ (ratio of hinge length to minimum thickness) are plotted. The influence of main parameters on stress concentration factor is studied and expressions of stress concentration factors are fitted by using finite element analysis results. The stiffness equations and maximum stress predicted by stress concentration factor equations are verified with finite element simulations. Fatigue life of CFLSFH is researched and the case results indicate that fillet can improve the fatigue life effectively.

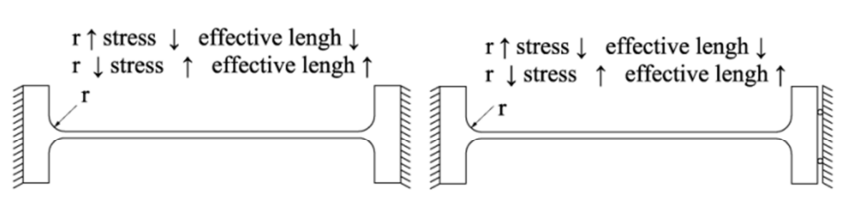

(a)

(b)

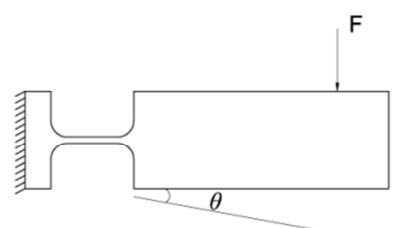

(c)

Figure 2. (a) Fixed-fixed CFLSFH (b) Fixed-guided CFLSFH (c) Corner filleted flexure hinge.

\section{Stiffness of CFLSFH}

According to Castigliano's theorem [32], the partial derivative of the strain energy of the elastomer with respect to load is equal to the corresponding displacement in the load direction, and it can be stated as:

$$
\Delta=\frac{\partial U}{\partial F}
$$

where $U$ is strain energy, $F$ is load, and $\Delta$ is the corresponding displacement.

When the external loading is applied to flexure hinges, the deformation in load direction can be calculated by Eq.(1). Generally, the leaf-spring type flexure hinge in actuators or sensors can be regarded as Euler-Bernoulli beams (the ratio of length to thickness is greater than 5), where shearing effects are ignored. Under this assumption, the flexible hinge has only bending deformation under the action of $F$, and the elastic strain energy is:

$$
U=\int \frac{M^{2} d x}{2 E I}
$$

where $M$ is bending moment, $E$ is the elastic modulus, $I$ is the moment of inertia.

The CFLSFH is shown in Figure 3, where $l, w, t$ and $r$ are the length, width, thickness and radius, respectively. Due to the corner-fillets, and thickness is varying with the coordinate $X$, which can be expressed as:

$$
t(x)=\left\{\begin{array}{l}
2\left(r-\sqrt{r^{2}-(r-x)^{2}}\right)+t_{0}, 0 \leq x \leq r \\
t_{0}, r<x<l-r \\
2\left(r-\sqrt{r^{2}-(x-l+r)^{2}}\right)+t_{0}, l-r \leq x \leq l
\end{array}\right.
$$



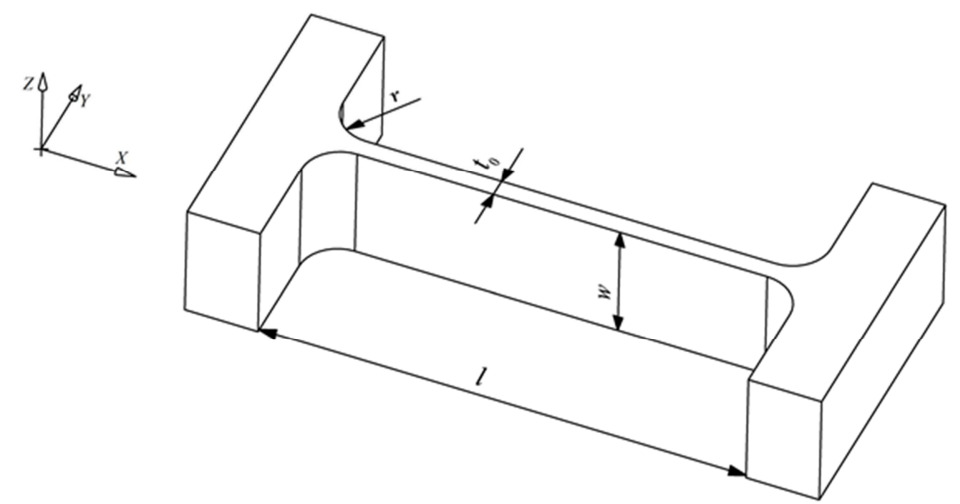

Figure 3. Geometric model of CFLSFH.

\subsection{Stiffness of Fixed-Guided CFLSFH}

The mechanics model of CFLSFH as guiding mechanism is shown in Figure 4, which has fixed-guided boundary conditions.

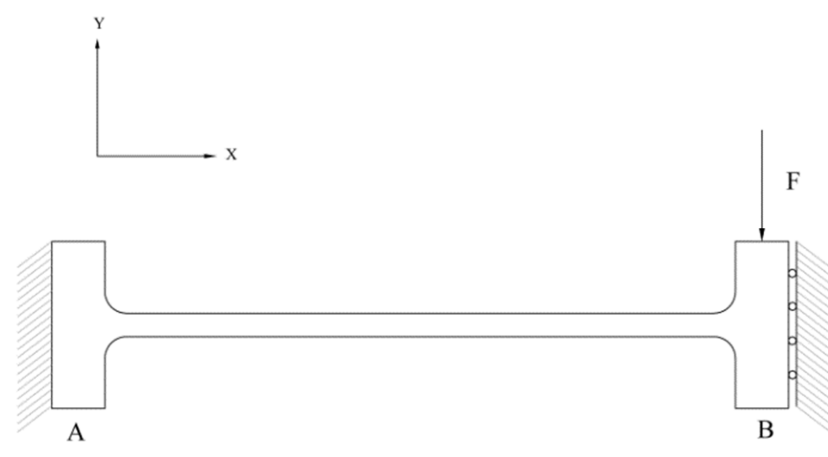

Figure 4. Mechanical model of fixed-guided CFLSFH.

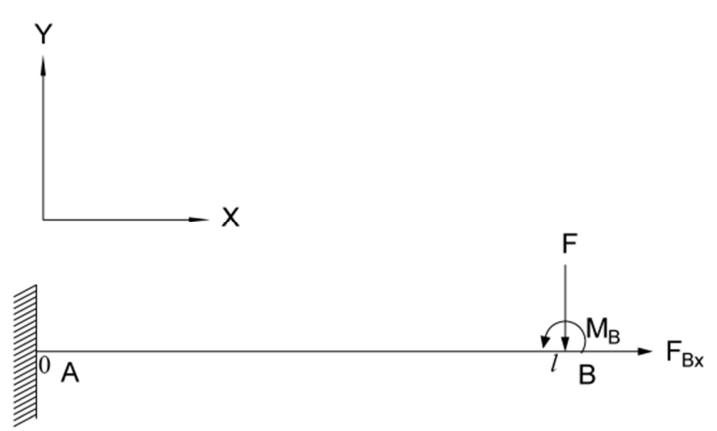

The fixed-guided CFLSFH is statically indeterminate to the second degree and therefore two compatibility equations will be necessary for the solution. Choosing support reactions at $\mathrm{B}$ as redundant and considering the compatibility of displacement, the flexure hinge then becomes statically determinate and stable. The equivalent statically model is shown in Figure 5.

The expression of bending moment along $X$ axis can be expressed as:

$$
M(x)=F\left(\frac{l}{2}-x\right), x \in[0, l]
$$

The elastic strain energy is obtained with Eq. (2) and Eq. (8). The displacement $\Delta_{g}$ of guided end point under the action of $F$ is obtained:

$$
\Delta_{f g}=\frac{\partial U}{\partial F}=\int_{l}^{12 F\left(\frac{l}{2}-x\right)^{2}} \frac{E w t^{3}(x)}{E x} d x
$$

Using dimensionless parameters $a$ and $b$, namely:

$$
\left\{\begin{array}{l}
a=r / t_{0} \\
b=l / t_{0}
\end{array}\right.
$$

and submitting them into Eq.(5) yields:

Figure 5. Statically model of fixed-guided CFLSFH.

$$
\Delta_{f g}=\frac{F}{E w}\left(\frac{6 \arctan (\sqrt{4 a+1}) G}{(2 a+1)(4 a+1)^{5 / 2}}+\frac{3 H}{2(2 a+1)(4 a+1)^{2}}+(b-2 a)^{3}\right)
$$

where

$$
\begin{aligned}
& G=96 a^{6}+128 a^{5}-80 a^{3}-50 a^{2}-12 a-1+24 a^{3} b^{2}-24 a^{3} b+6 a^{2} b^{2}-96 a^{5} b+24 a^{4} b^{2}-96 a^{4} b \\
& H=-160 a^{5}+32 a^{4} b+24 a^{3} b^{2}+32 \pi a^{3}-32 a^{4}+16 a^{2} b^{2}+32 \pi a^{2}+72 a^{3}-8 a^{2} b+4 a b^{2}+10 \pi a+32 a^{2}+\pi+4 a
\end{aligned}
$$

Finally, the guiding stiffness can be expressed as: 


$$
K_{f g}=\frac{F}{\Delta_{f g}}=2 E w /\left(\frac{6 \arctan (\sqrt{4 a+1}) G}{(2 a+1)(4 a+1)^{5 / 2}}+\frac{3 H}{2(2 a+1)(4 a+1)^{2}}+(b-2 a)^{3}\right)
$$

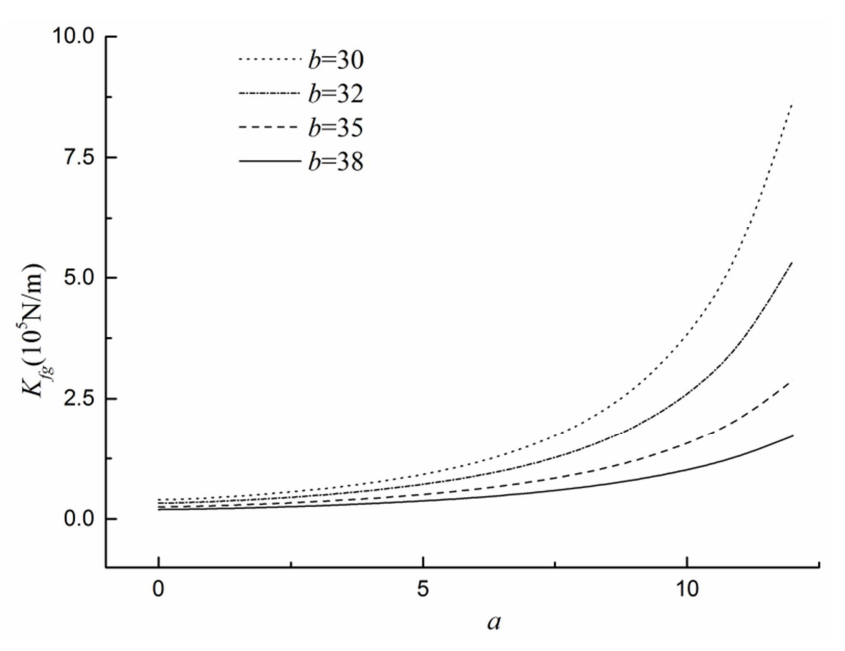

(a)

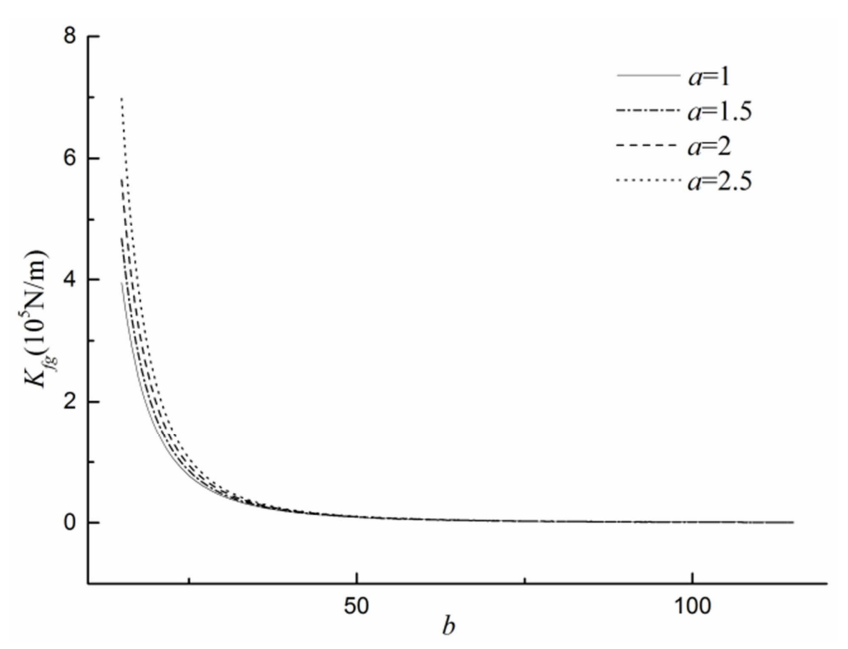

(b)

Figure 6. Variation of stiffness $K_{f g}$ of fixed-guided CFLSFH with dimensionless geometric parameters. (a) Variationof $K_{f g}$ with a; (b) Variation of $K_{f g}$ with $b$.

Figure 6 shows variation of stiffness of fixed-guided CFLSFH with dimensionless geometric parameters. Figure 6(a) indicates that the increase of value of $a$ strengthens the stiffness of fixed-guided CFLSFH, while the increase of value of $b$ slows this trend down. Figure 6(b) demonstrates that the increase of value of $b$ weakens the stiffness of CFLSFH, and the influence of $a$ to stiffness is ignorable when $b$ increases to 40 .

\subsection{Stiffness of Fixed-Fixed CFLSFH}

The mechanics model of fixed-fixed CFLSFH shown in Figure 7, is subjected to concentrated load $F$ at the midpoint.

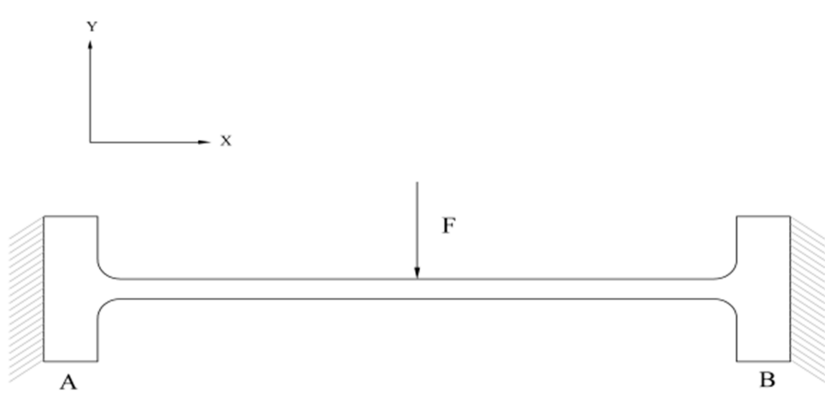

Figure 7. Mechanical model of fixed-fixed CFLSFH.
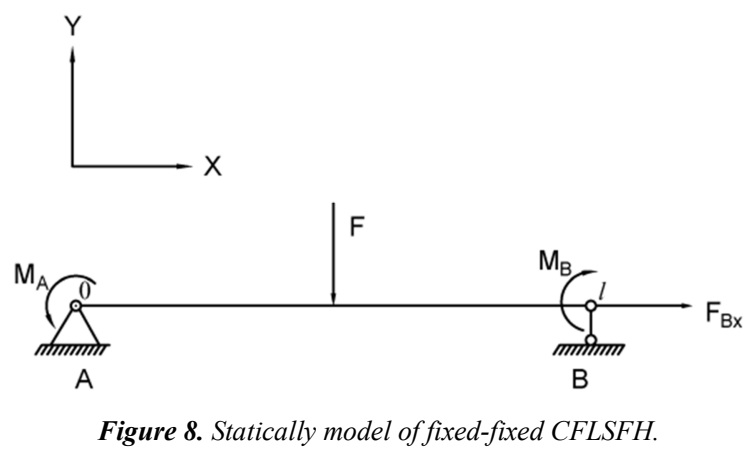

The fixed-fixed CFLSFH is statically indeterminate to the third degree. Releasing redundant constrains at A and B and considering the compatibility of displacement, statically indeterminate problem can be converted to a statically determinate problem. The equivalent statically model is shown in Figure 8.

The moment formulation along $X$ axis direction can be expressed as:

$$
M(x)=\left\{\begin{array}{l}
\frac{F x}{2}-\frac{F c t_{0}}{8}, x \in\left[0, \frac{l}{2}\right] \\
\frac{F l}{2}-\frac{F x}{2}-\frac{F c t_{0}}{8}, x \in\left[\frac{l}{2}, l\right]
\end{array}\right.
$$

where $c=\frac{(2 a+1)(A-B)}{C-D}$

$$
\begin{gathered}
A=\sqrt{4 a+1}\left(64 a^{4}+40 a^{3}-b^{2}(4 a+1)^{2}\right) \\
B=48 a^{3} \arctan \sqrt{4 a+1}(2 a+1) \\
C=\sqrt{4 a+1}\left(64 a^{4}+52 a^{3}+12 a^{2}-b(2 a+1)(4 a+1)^{2}\right) \\
D=12 a^{2} \arctan \sqrt{4 a+1}(2 a+1)^{2}
\end{gathered}
$$

The deflection of CFLSFH based on Castigliano's theorem and symmetry principle is shown in Eq. (10). 


$$
\begin{gathered}
\Delta_{f f}=\frac{\partial U}{\partial F}=2 \int_{0}^{\frac{l}{2}} \frac{F\left(\frac{x}{2}-\frac{c t_{0}}{8}\right)^{2}}{E I(x)} d x \quad \text { Computing the integral yields: } \\
\Delta_{f f}=\frac{F}{E w}\left(\frac{3 I \arctan \sqrt{4 a+1}}{4(2 a+1)(4 a+1)^{5 / 2}}+\frac{3 J}{8(2 a+1)(4 a+1)^{2}}+2\left(\frac{b}{2}-\frac{c}{4}\right)^{3}-2\left(a-\frac{c}{4}\right)^{3}\right)
\end{gathered}
$$

where

$$
\begin{aligned}
& I=3 a^{2} c^{2}(2 a+1)^{2}-24 a^{3} c(2 a+1)^{2}+192 a^{6}+256 a^{5}-160 a^{3}-100 a^{2}-24 a-2 \\
& J=\left(6 a^{2}+4 a+1\right) a c^{2}+(2 a+1)(4 a+1)^{2} \pi-4 a\left(40 a^{4}+8 a^{3}-18 a^{2}-8 a-1\right)+4 a^{2} c\left(4 a^{2}-1\right)
\end{aligned}
$$

Stiffness of fixed-fixed CFLSFH can be thus expressed as:

$$
K_{f f}=\frac{F}{\Delta_{f f}}=E w /\left(\frac{3 I \arctan \sqrt{4 a+1}}{4(2 a+1)(4 a+1)^{5 / 2}}+\frac{3 J}{8(2 a+1)(4 a+1)^{2}}+2\left(\frac{b}{2}-\frac{c}{4}\right)^{3}-2\left(a-\frac{c}{4}\right)^{3}\right)
$$

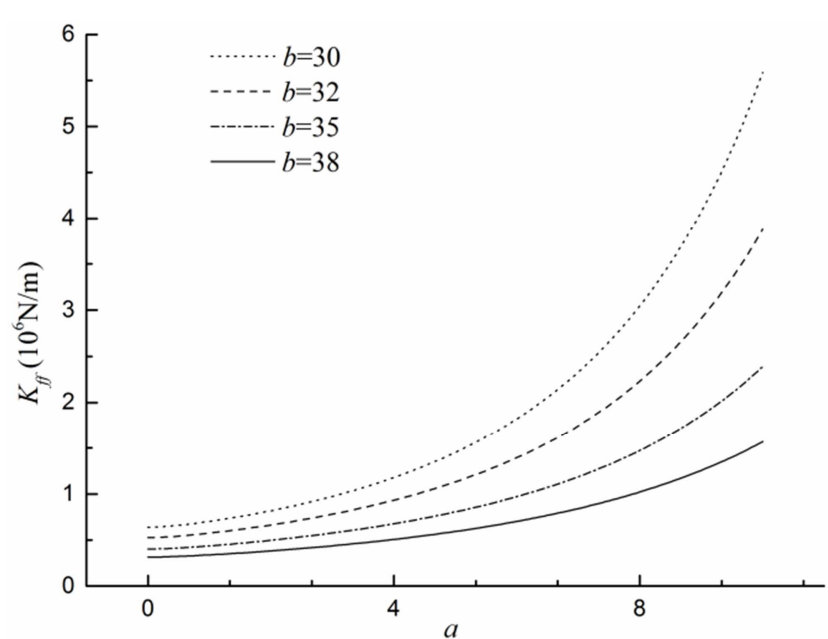

(a)

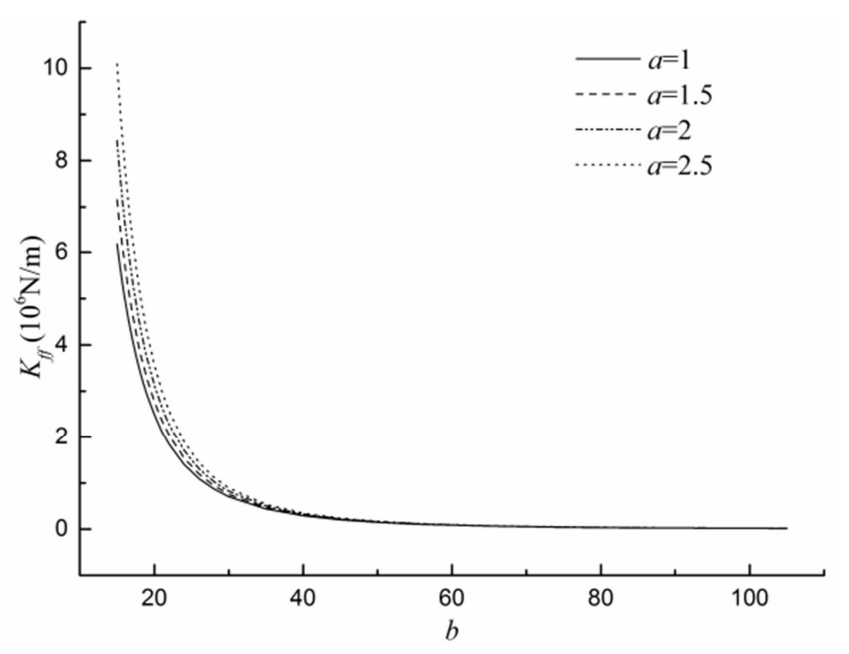

(b)

Figure 9. Variation of stiffness with parameters for fixed-fixed CFLSFH. (a) Variation of $K_{f f}$ with parameter a; (b) Variation of $K_{f f}$ with parameter $b$.

Variation of stiffness of fixed-fixed CFLSFH with parameter is plotted in Figure 9. Figure 9(a) indicates that increasing of the value of $a$ strengthens the stiffness of fixed-fixed CFLSFH, while increasing of the value of $b$ slows the increase trend of stiffness down. Figure 9(b) illustrates that increasing of the value of $b$ weakens the stiffness of CFLSFH, and the influence of $a$ on stiffness is neglectable when $b$ increase to 40 .

\section{Stress Concentration Factors}

The local material reduction of flexible hinge causes an increase of local stress, and the actual maximum stress of flexure hinge is much higher than the predictions of mechanics of materials equations. Stress concentration factor is used to characterize the increase of stress, which is defined as the ratio of actual maximum stress to normal maximum stress. Therefore, stress concentration factor can be used to estimate actual maximum stress of flexure hinge in the design of compliant mechanism, which can be expressed as [34]:

$$
k=\frac{\sigma_{\max }}{\sigma_{\text {nom }}}
$$

where $\sigma_{\max }$ is actual maximum bending stress, $k$ denotes stress concentration factor and $\sigma_{\text {nom }}$ is normal maximum stress.

\subsection{Stress Concentration Factor of Fixed-Guided CFLSFH}

For fixed-guided CFLSFH, stress concentration occurs in the connection positions of fillet with straight beam. The moment of fixed-guided CFLSFH in stress concentration part can be evaluated using Eq. (4), and the normal maximum stress can be expressed as:

$$
\sigma_{\text {nom }}^{f f}=\frac{M}{I} y_{\max }=\frac{3 F(l-2 r)}{w t_{0}^{2}}=\frac{3 F(b-2 a)}{A}
$$


where $y_{\max }$ represents the maximum distance to the neutral axis of the cross section, $A$ is the sectional area of minimum cross section.

COMSOL software are used to do with FEA of flexure hinges. Ninety models are generated to provide abundant stress results for curve fitting. The main geometric parameters of flexure hinge are $1 \mathrm{~mm} \leq t \leq 2 \mathrm{~mm}, 0.2 \leq a \leq 5,40 \leq b \leq 100$. The depth of modeled flexure hinges is $10 \mathrm{~mm}$ with a Young's modulus (E) of 71.7Gpa and a Poisson ratio (v) of 0.32 . Hexahedral element is chosen to generate the model mesh, which is more accurate than triangle element. Each model is fixed on one end, and roller constraint is added on the other end. The load is applied in the roller end. The maximum stress of every analysis are recorded to calculate stress concentration factor.

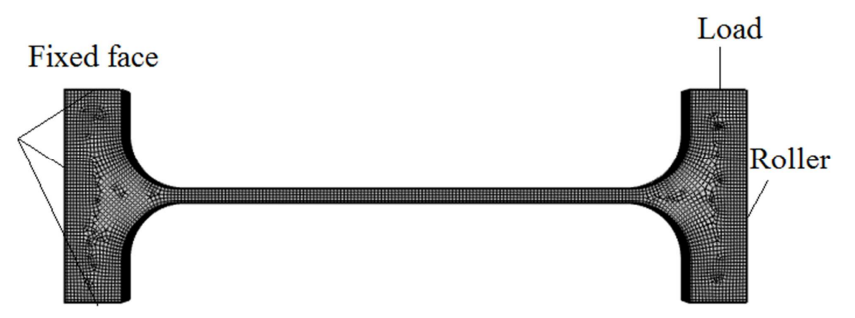

Figure 10. FEA model of fixed-guided.

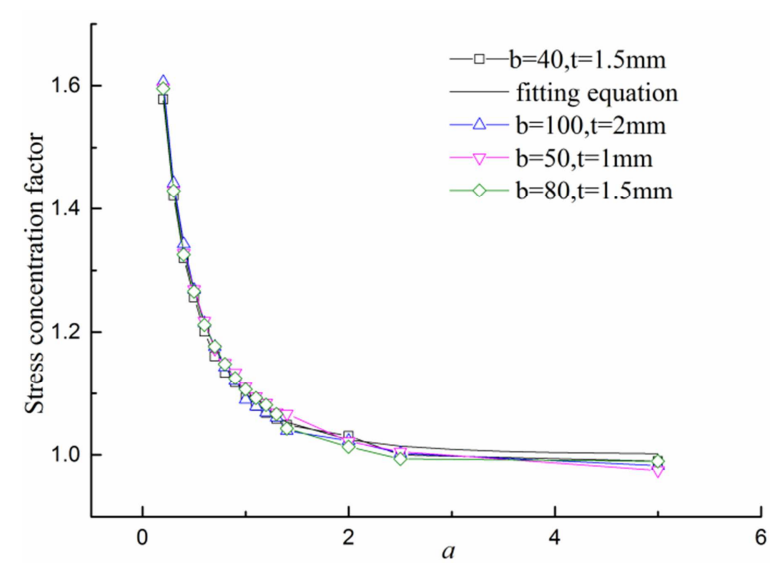

Figure 11. Stress concentration factor for fixed-guided CFLSFH.

The FEA results confirm that the stress concentration factors are dominated by non-dimensional parameter $a$, while the parameter $b$ has negligible effects on stress concentration factors, as Figure 11 shows. The empirical equation of guiding stress concentration factor $k^{f g}$ is obtained by fitting the results of FEA, as Eq.(15) shows.

$$
k^{f g}=1+\left(\frac{1.095}{a+1.0765}\right)^{3.6}
$$

According to Figure 11 (b), stress concentration factors are sharply reduced with the increase of $a$. Stress concentration factors are approximately equal to 1 , when $a$ is in the range of 2 to 3 . Therefore, $[2,3]$ is the optimal range of $a$, synthetically considering the stiffness and stress concentration.

\subsection{Stress Concentration Factor of Fixed-Fixed CFLSFH}

The moment of fixed-fixed CFLSFH in stress concentration part can be obtained according to Eq. (9). The maximum nominal stress is expressed as:

$$
\sigma_{\text {nom }}^{f f}=\frac{M}{I} y_{\max }=\frac{3 F\left(c t_{0}-4 r\right)}{4 w t_{0}{ }^{2}}=\frac{3 F(c-4 a)}{4 A}
$$

The analysis models of fixed-fixed CFLSFH is built using the method similarly to section 3.1. The parameter of models are: $1 \mathrm{~mm} \leq t_{0} \leq 2 \mathrm{~mm}, w=15 \mathrm{~mm}, 0.2 \leq a \leq 5,40 \leq b \leq 80$. Model of each design is meshed with hexahedral element. Each model is fixed on the both end, and a unit load is loaded at the midpoint of CFLSFH. FEA results for $k$ is calculated using Eq. (13), based on maximum stress of FEA.

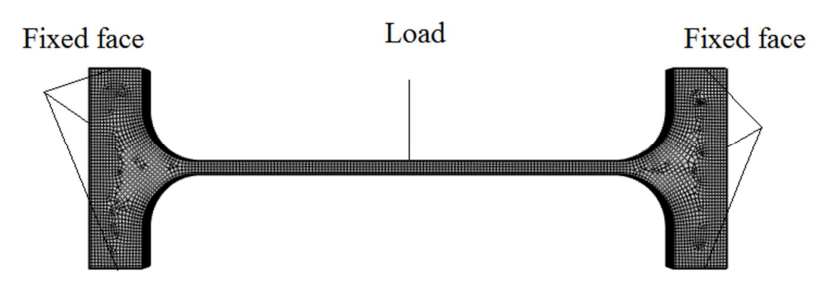

Figure 12. FEA model of fixed-fixed CFLSFH.

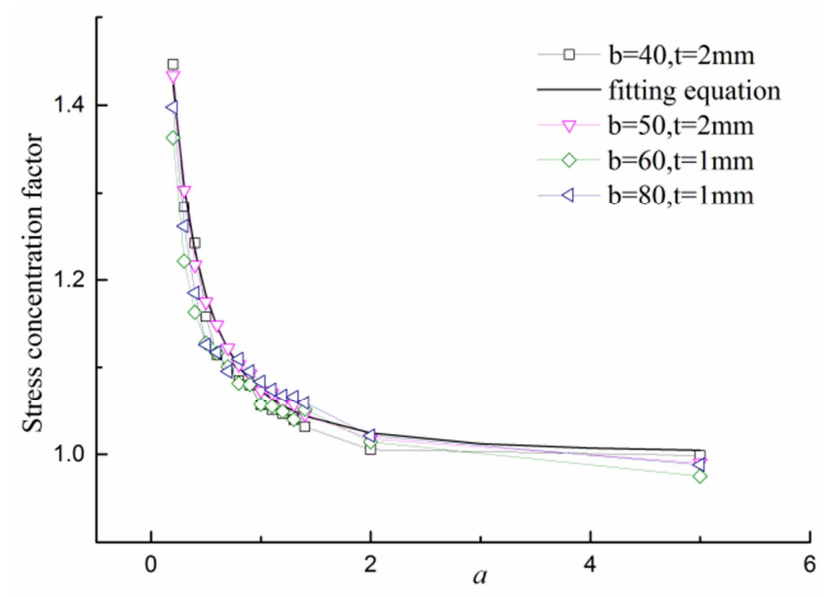

Figure 13. Stress concentration factor for fixed-fixed CFLSFH.

The FEA results confirm that the stress concentration factors are dominated by non-dimensional parameter $a$, while the parameter $b$ has negligible effects on stress concentration factors, as Figure 13 shows. The following empirical equations for $k^{f f}$ is obtained by fitting the FEA results.

$$
k^{f f}=1+\left(\frac{0.385}{a+0.387}\right)^{2.04}
$$

According to Figure 13 (b), stress concentration factors are sharply reduced with the increase of $a$. Stress concentration factors are approximately equal to 1 , when $a$ is in the range of 2 to 3 . 


\section{Verification}

The COMSOL software has been utilized to calculate stiffness and stress for the design cases listed in Table 1 . The material parameters of models are: $E=71.7 \mathrm{GPa}, \mu=0.32$, $\rho=2810 \mathrm{Kg} / \mathrm{m}^{3}$. The models built method is same to which is shown in Section 3. Hexahedral element is chosen for higher accuracy. The load $\mathrm{F}$ applied on flexure hinge is $1 \mathrm{~N}$.

Table 2 and Table 3 comprises the FEA results and analytical results together with the design parameters of several CFLSFHs in different constrains. The analytical results and FEA results are in good agreement. Compared to FEA results, the maximum errors of stiffness is $2.32 \%$, and the maximum errors of stress is $1.44 \%$.

Table 1. Geometric parameters of CFLSFHs.

\begin{tabular}{|c|c|c|c|c|c|c|c|c|}
\hline \multirow{2}{*}{ Case NO. } & \multicolumn{4}{|c|}{ Fixed-guided CFLSFH } & \multicolumn{4}{|c|}{ Fixed-fixed CFLSFH } \\
\hline & $l(\mathrm{~mm})$ & $r(\mathrm{~mm})$ & $t(\mathrm{~mm})$ & $w(\mathrm{~mm})$ & $l(\mathrm{~mm})$ & $r(\mathbf{m m})$ & $t(\mathrm{~mm})$ & $w(\mathbf{m m})$ \\
\hline 1 & 40 & 2 & 2 & 15 & 80 & 2 & 2 & 15 \\
\hline 2 & 40 & 4 & 2 & 15 & 80 & 4 & 2 & 15 \\
\hline 3 & 40 & 6 & 2 & 15 & 80 & 6 & 2 & 15 \\
\hline 4 & 40 & 8 & 2 & 15 & 80 & 8 & 2 & 15 \\
\hline
\end{tabular}

Table 2. Results of Fixed-guided CFLSFHs.

\begin{tabular}{|c|c|c|c|c|c|c|}
\hline \multirow{2}{*}{ Case NO. } & \multicolumn{3}{|l|}{ Stiffness } & \multicolumn{3}{|l|}{ Stress } \\
\hline & Analytical(N/m) & FEA(N/m) & Error (\%) & Eqs. $\left(10^{6} \mathrm{~N} / \mathrm{m}^{2}\right)$ & $\operatorname{FEA}\left(10^{6} \mathrm{~N} / \mathrm{m}^{2}\right)$ & Error (\%) \\
\hline 1 & 155619 & 152439 & 2.09 & 1.932 & 1.96 & -1.44 \\
\hline 2 & 197152 & 194932 & 1.14 & 1.639 & 1.64 & -0.08 \\
\hline 3 & 260725 & 258398 & 0.90 & 1.417 & 1.40 & 1.18 \\
\hline 4 & 358865 & 354610 & 1.20 & 1.208 & 1.20 & 0.87 \\
\hline
\end{tabular}

Table 3. Results of Fixed-fixed CFLSFHs.

\begin{tabular}{|c|c|c|c|c|c|c|}
\hline \multirow{2}{*}{ Case NO. } & \multicolumn{3}{|l|}{ Stiffness } & \multicolumn{3}{|l|}{ Stress } \\
\hline & Analytical (N/m) & FEA(N/m) & Error (\%) & Eqs. $\left(10^{5} \mathrm{~N} / \mathrm{m}^{2}\right)$ & FEA $\left(10^{5} \mathrm{~N} / \mathrm{m}^{2}\right)$ & Error (\%) \\
\hline 1 & 289032 & 282486 & -2.32 & 9.92 & 9.80 & 1.23 \\
\hline 2 & 323697 & 317460 & -1.96 & 8.81 & 8.72 & 0.01 \\
\hline 3 & 367962 & 361011 & -1.93 & 8.10 & 7.91 & 0.02 \\
\hline 4 & 422918 & 414938 & -1.92 & 7.47 & 7.33 & 0.02 \\
\hline
\end{tabular}

\section{Fatigue Life of CFLSFH}

Fatigue can lead to premature failure of flexure hinge, especially in high frequency and high speed operational situations. Therefore fatigue life is important performance for the vulnerable flexure hinges. The fillet can reduce the stress of CFLSFH and prolong its fatigue life. Stress-life method is used to research the relationship between fillet radius and fatigue life. The fatigue strength of smooth test specimen for Aluminum alloys can be estimate by the following equation [34].

$$
S_{e}= \begin{cases}0.4 S_{U} & S_{U} \leq 325 \mathrm{MPa} \\ 130 \mathrm{MPa} & S_{U} \geq 325 \mathrm{MPa}\end{cases}
$$

where $S_{U}$ is the ultimate tensile strength and $S_{e}$ is the fatigue strength of material.

The fatigue strength of flexure hinge with no mean stress which account for notch effect, surface finish and preload type, is defined as

$$
S_{e}^{\prime}=k_{a} k_{b} k_{c} S_{e}
$$

where $k_{a}$ is the fatigue stress concentration factor, $k_{b}$ is the surface condition modification factor, and $k_{c}$ is load modification factor.

The fatigue stress concentration factor can be calculated as

$$
\frac{1}{k_{a}}=1+\frac{k_{t}-1}{1+A_{P} / r}
$$

where $k_{t}$ is stress concentration factor, $A_{P}$ is material constants, for Aluminum alloys $A_{P} \approx 0.66$, and $r$ is the radius of curvature.

The surface condition modification factor can be estimated according the surface roughness, which is proposed in [21]. The load factor is defined as

$$
k_{c}= \begin{cases}1 & \text { bending } \\ 0.85 & \text { axial } \\ 0.59 & \text { torsion }\end{cases}
$$

The fatigue safety factor is calculated as

$$
S_{D}=\frac{S_{e}^{\prime}}{\sigma_{m}}
$$

where $\sigma_{m}$ is normal stress.

Then the fatigue safety factor of CFLSFH in fixed-guided and fixed-fixed condition can be expressed as 


$$
S_{D}= \begin{cases}\frac{k_{b} k_{c} S_{e} A}{3 F(b-2 a)\left(1+\frac{k^{f g}-1}{1+A_{P} / r}\right)} & \text { for fixed-guided CFLSFH } \\ \frac{4 k_{b} k_{c} S_{e} A}{3 F(c-4 a)\left(1+\frac{k^{f f}-1}{1+A_{P} / r}\right)} & \text { for fixed - fixed CFLSFH }\end{cases}
$$

A case is taken to illustrate the influence of fillet radius to fatigue life of CFLSFH. Consider a given CFLSFH $\left(\mathrm{t}_{0}=4 \mathrm{~mm}\right.$, $\mathrm{w}=15 \mathrm{~mm}$ ) is undergoing a cycling load $\mathrm{F}= \pm 100 \mathrm{~N}$. The compliance mechanism is made of $7075 \mathrm{~T} 6$ and manufactured by $\mathrm{CNC}$ milling process (surface roughness $\mathrm{R}=6.3 \mu \mathrm{m}$ ). The ultimate tensile strength is larger than $325 \mathrm{MPa}$, therefore $S_{e}$ is $130 \mathrm{MPa} . k_{b}$ can be estimated according surface roughness and is 0.87 . Figure 14 shows that the increase of fillet radius can improve the fatigue life of flexure hinge effectively. In the meantime, boundary condition have obvious influence on the fatigue life of flexure hinge.

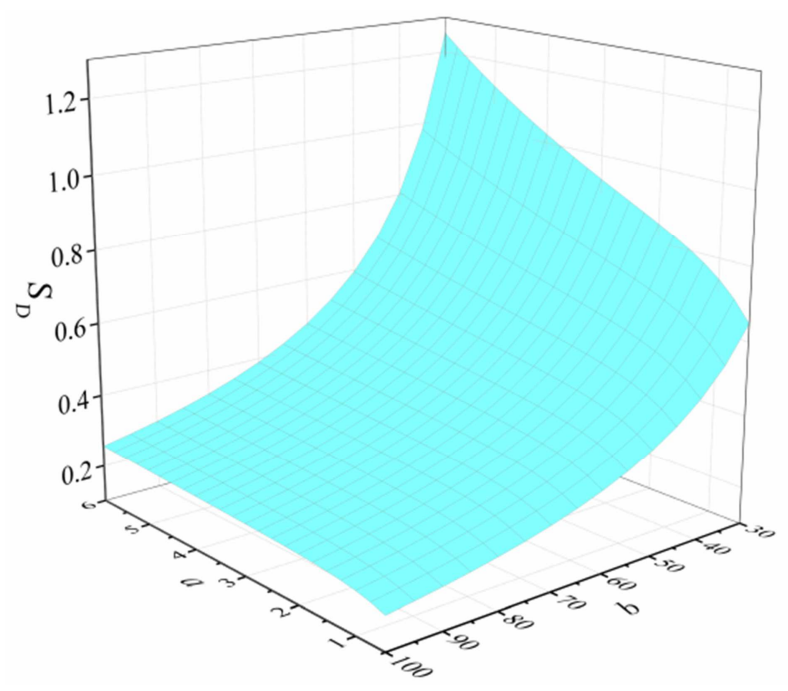

(a) Fixed-guided condition

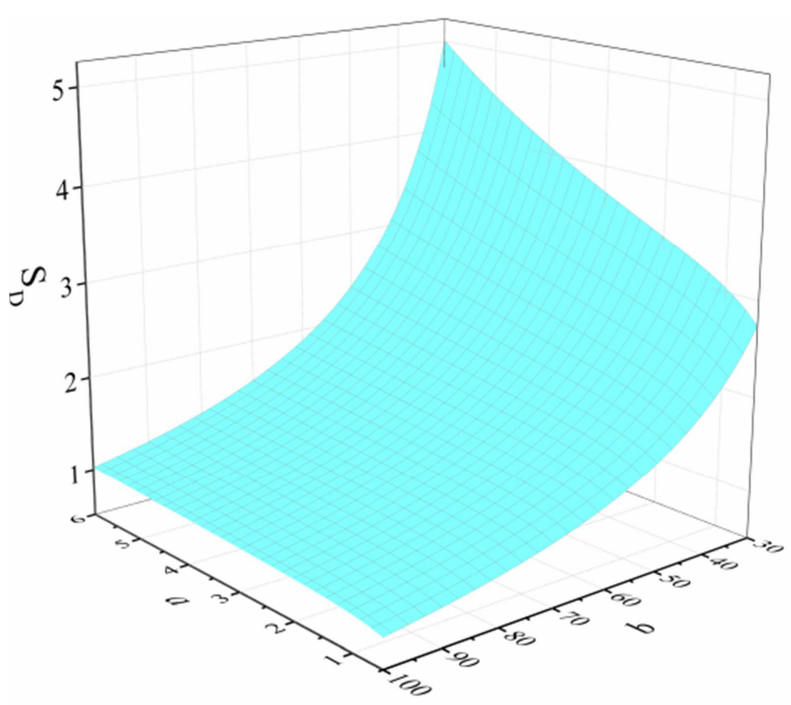

(b) Fixed-Fixed condition

Figure 14. Fatigue safety factor of CFLSFHs.

\section{Conclusions}

This paper proposes analytic solutions of stiffness of CFLSFH in fixed-fixed and fixed-guided boundary condition, which are widely used in micro-position stages and sensors. The stiffness equations are derived based on Castigliano's theorem and force method. Stress concentration factors of CFLSFHs are investigated according to the analytical results of COMSOL software. Parameter $a$ (ratio of radius to minimum thickness) is verified to be the main influence factor to stress concentration and equations of stress concentration factors are fitted taking $a$ as fitted variable. By confirming stiffness equations and equations of stress concentration factors with COMSOL software, the stiffness errors are less than $3 \%$, and stress errors are less than $2 \%$. Case results indicate that fillet radius can improve fatigue life of CFLSFH effectively. Analytical results of CFLSFHs can be used for the design and further optimization of CFLSFH based mechanism.

\section{Acknowledgements}

This work was supported by the National Natural Science Foundation of China (Grant Nos. 91648108, U1601202, 51675106), Guangdong Natural Science Foundation (Grant Nos. 2015A030312008, 2015A030308004), Guangdong Science and Technology Plan (Grant Nos. 2015B010104006, 2015B010104008, 2013B010402014), and Science and Technology Program of Guangzhou (Grant No. 201510010281).

\section{References}

[1] Chen X, Su C Y, Li Z, et al., Design of Implementable Adaptive Control for Micro/Nano Positioning System Driven by Piezoelectric Actuator. IEEE Transactions on Industrial Electronics, 2016, 63(10):6471-6481.

[2] S. B. Choi, S. S. Han, Y. M. Han, et al., A magnification device for precision mechanisms featuring piezoactuators and flexure hinges: Design and experimental validation. Mechanism and Machine Theory, 2007. 42(9): p. 1184-1198.

[3] W. Dong, J. Tang, and Y. ElDeeb, Design of a linear-motion dual-stage actuation system for precision control. Smart Materials \& Structures, 2009. 18(9).

[4] Akbari S and Pirbodaghi T, Precision positioning using a novel six axes compliant nano-manipulator. Microsystem Technologies, 2016, 23(7):1-9.

[5] Sun X and Yang B, A new methodology for developing flexure-hinged displacement amplifiers with micro-vibration suppression for a giant magnetostrictive micro drive system, Sensors \& Actuators A Physical, 2017, 263.

[6] Zhang $X$ and Xu Q, Design and testing of a new 3-DOF spatial flexure parallel micropositioning stage. International Journal of Precision Engineering \& Manufacturing, 2018, 19(1):109-118.

[7] Qu J, Chen W, Zhang J, et al., A piezo-driven 2-DOF compliant micropositioning stage with remote center of motion. Sensors \& Actuators A Physical, 2016, 239:114-126. 
[8] Wang F, Liang C, Tian Y, et al., A Flexure-Based Kinematically Decoupled Micropositioning Stage With a Centimeter Range Dedicated to Micro/Nano Manufacturing. IEEE/ASME Transactions on Mechatronics, 2016, 21(2):1055-1062.

[9] Q. Xu, Design and Development of a Flexure-Based Dual-Stage Nanopositioning System With Minimum Interference Behavior. IEEE Transactions on Automation Science and Engineering, 2012. 9(3): p. 554-563.

[10] Zhang Y, Zhang W, Zhang Y, et al., 2-D Medium-High Frequency Fiber Bragg Gratings Accelerometer. IEEE Sensors Journal, 2017, 17(3):614-618.

[11] S. Desrochers, D. Pasini, and J. Angeles, Optimum Design of a Compliant Uniaxial Accelerometer. Journal of Mechanical Design, 2010. 132(4).

[12] S. Kavitha, R. J. Daniel, and K. Sumangala, A simple analytical design approach based on computer aided analysis of bulk micromachined piezoresistive MEMS accelerometer for concrete SHM applications. Measurement, 2013. 46(9): p. 3372-3388.

[13] Chen D, Wu Z, Shi X, Wang J, Liu L, Design and modelling of an electromagnetically excited silicon nitride beam resonant pressure sensor, In: Proceedings 4th IEEE international conference nano/micro engineered and molecular systems, 2009, pp.754-757

[14] M. J. Lachut and J. E. Sader, Effect of surface stress on the stiffness of thin elastic plates and beams. Physical Review B, 2012. 85(8).

[15] D. Kang, et al., Optimal design of high precision XY-scanner with nanometer-level resolution and millimeter-level working range. Mechatronics, 2009. 19(4): p. 562-570. DOI: 10.1016/j.mechatronics.2009.01.002

[16] Q. Xu, Design, testing and precision control of a novel long-stroke flexure micropositioning system. Mechanism and Machine Theory, 2013. 70: p. 209-224.

[17] G. Chen, X. Y. Liu, H. Gao, A generalized model for conic flexure hinges. Review of Scientific Instruments, 2009. 80(5).

[18] N. Lobontiu, J. S. N. Paine and E. Garcia, et al., Design of symmetric conic-section flexure hinges based on closed-form compliance equations. Mechanism and Machine Theory, 2002. 37(5): p. 477-498.

[19] G. M. Chen, X. Shao, and X. Huang, A new generalized model for elliptical arc flexure hinges. Review of Scientific Instruments, 2008. 79(9).

[20] Y. Tian, B. Shirinzadeh, and D. Zhang, Closed-form compliance equations of filleted V-shaped flexure hinges for compliant mechanism design. Precision Engineering-Journal of the International Societies for Precision Engineering and Nanotechnology, 2010. 34(3): p. 408-418.

[21] F. Dirksen, M. Anselmann and TI. Zohdi, Incorporation of flexural hinge fatigue-life cycle criteria into the topological design of compliant small-scale devices. Precision Engineering-Journal of the International Societies for Precision Engineering and Nanotechnology, 2013. 37(3): p. 531-541.

[22] Q. Wang, and X. M. Zhang, Fatigue reliability based optimal design of planar compliant micropositioning stages. Review of Scientific Instruments, 2015. 86(10):p.105-117.

[23] Y. M. Tseytlin, Notch flexure hinges: An effective theory. Review of Scientific Instruments, 2002. 73(9): p. 3363-3368.

[24] Y. F. Wu, and Z. Y. Zhou, Design calculations for flexure hinges. Review of Scientific Instruments, 2002. 73(8): p. 3101-3106.

[25] Y. K. Yong, T. F. Lu, and D. C. Handley, Review of circular flexure hinge design equations and derivation of empirical formulations. Precision Engineering-Journal of the International Societies for Precision Engineering and Nanotechnology, 2008. 32(2): p. 63-70.

[26] Y. K. Yong, T. F. Lu, Comparison of circular flexure hinge design equations and the derivation of empirical stiffness formulations, in 2009 IEEE/ASME International Conference on Advanced Intelligent Mechatronics, 2009. p. 510-515.

[27] G. Chen, J. Wang, and X. Liu, Generalized Equations for Estimating Stress Concentration Factors of Various Notch Flexure Hinges. Journal of Mechanical Design, 2014. 136(3).

[28] N. Lobontiu, et al., Stiffness characterization of corner-filleted flexure hinges. Review of Scientific Instruments, 2004. 75(11): p. $4896-4905$.

[29] Z. Yang, Y. Bai, X. Chen, Simultaneous optimal design of topology and size for a flexure-hinge-based guiding mechanism to minimize mass under stiffness and frequency constraints. Engineering Optimization, 2017.49(6):p. 948-961.

[30] N. Lobontiu, J. S. N Paine, E. Garcia, et al., Corner-filleted flexure hinges. Journal of Mechanical Design, 2001. 123(3): p. 346-352.

[31] Q. Meng, Y. Li, and J. Xu, New empirical stiffness equations for corner-filleted flexure hinges. Mechanical Sciences, 2013. 4(2): p. 345-356.

[32] F. P. Beer, Mechanics of Materials, McGraw Hill, New York, 2012.

[33] W. Young, R Budynas, Sadegh A. Roark's Formulas for Stress and Strain, seventh ed., McGraw Hill, New York, 2002.

[34] R. C. Juvinall. Stress, Strain, and Strength., McGraw Hill, New York, 1967. 